\title{
МАКРОЭКОНОМИЧЕСКОЕ РАВНОВЕСИЕ В «ОТКРЫТОЙ ЭКОНОМИКЕ»: МОДЕЛЬ Р.А. МАНДЕЛЛА
}

\section{(c) 2019 Лихачев Михаил Олегович}

доктор экономических наук, доцент, профессор кафедры Экономической теории и менеджмента Московский педагогический государственный университет, Россия, Москва

E-mail: olegmix71@mail.ru

В статье рассматриваются базовые принципы и теоретическая архитектура модели «открытой экономики» Р.А. Манделла, которая основывается на гибком сочетании кейнсианских и неоклассических принципов макроэкономического анализа. Автор рассматривает условия стабильного равновесия в модели Р.А. Манделла и возможности его применения к анализу стабильности национальной экономики в современных условиях.

Ключевые слова: макроэкономика, «открытая экономика», макроэкономическое равновесие, модель Р.А. Манделла, макроэкономическая политика, бюджетно-налоговая политика, денежно-кредитная политика, макроэкономическая стабильность

Теория равновесия в открытой экономике является закономерным результатом развития общей теории макроэкономического равновесия. Можно сказать, что теория равновесия в закрытой экономике, разрабатывавшаяся в экономической науке в течение долгого времени была лишь подготовительным этапом к созданию более общей теории, охватывающей не только внутренние взаимосвязи экономической системы, но и связи национальной экономики с внешним миром. Модель закрытой экономики была необходимой теоретической абстракцией, которая позволяла упростить анализ экономической системы с тем, чтобы, сформулировать основные принципы теории. В процессе дальнейшего развития концепции макроэкономического равновесия исследователи, неизбежно, сталкивались с необходимостью приблизить теоретическую модель к действительности, введя в нее параметры, описывающие взаимодействие национальной экономики с внешним миром.

Краткосрочное равновесие в открытой экономике рассматривается как согласование двух равновесных состояний - внутреннего и внешнего. Внутреннее равновесие предполагает согласование спроса и предложения на товары национального производства, а также объемов сбережений и инвестиций, денежной массы, уровня цен и ставки процента. А внешнее равновесие предполагает достижение нулевого сальдо платежного баланса при соответствующем равновесном значении валютного курса.
Параметры внутреннего и внешнего равновесия взаимосвязаны между собой и оказывают взаимное влияние друг на друга. Во-первых, спрос на товары национального производства включает в себя внешнюю компоненту в виде величины чистого экспорта, который также может рассматриваться как сальдо счета текущих операций в платежном балансе. Величина чистого экспорта определяется «условиями торговли» или реальным курсом национальной валюты, который определяется отношением внутренних и внешних цен при данном уровне обменного курса национальной валюты. Повышение реального курса национальной валюты за счет роста номинального курса или внутреннего уровня цен приводит к сокращению чистого экспорта, а его снижение, наоборот, - к увеличению.

Во-вторых, величина внутренней ставки процента в соотношении с ее среднемировым уровнем, определяет потоки капитала из страны за границу и из-за границы в страну, что неизбежно отражается на величине сальдо счета движения капиталов в платежном балансе. Превышение внутренней ставки процента над ее среднемировым уровнем, вызывает увеличение притока капитала из-за границы и рост сальдо счета движения капиталов, а снижение внутренней ставки процента ниже среднемирового уровня усиливает отток капитала из страны и способствует снижению величины сальдо счета движения капитала.

Исходя из этого, условия внутреннего рав- 
новесия можно рассматривать как соблюдение условий тождества национальных счетов ех ante (a не ex post, как это описывает традиционная макроэкономическая статистика. Тогда совокупное предложение будет изначально соответствовать объему совокупного спроса, а не адаптироваться к нему в результате коррекции объемов производства и уровня цен.

Условия внешнего равновесия можно представить как разность чистого экспорта товаров и услуг и чистого экспорта капитала - сальдо счета движения капитала в платежном балансе. То есть внешнее равновесие означает идеальное состояние платежного баланса, в котором нулевое сальдо достигается не как результат манипуляций Центрального банка со своими официальными резервами, а как стихийное балансирование денежных потоков, идущих из страны за границу и из-за границы в страну.

Одной из первых моделей равновесия в «открытой экономике» была модель Р.А. Манделла, в рамках которой совмещались кейнсианские представления о роли эффективного спроса в определении объемов производства, уровня занятости и доходов национальной экономики и неклассические представления о стремлении экономической системы к равновесию, совместимому с полным использованием ресурсов, в том числе и с полной занятостью $[1 ; 2 ; 4 ; 7 ; 8]$. В современных условиях, когда конфликт между неоклассическими и кейнсианскими принципами макроэкономического анализа обрел новую остроту, анализ базовых принципов и исходных моделей этих теоретических направлений снова становится актуальным.

\section{Модель Р.А. Манделла}

Р.А. Манделл рассматривает экономическую систему, стремящуюся к одновременному достижению внутреннего и внешнего равновесия за счет корректировки двух основных параметров - процентной ставки и реального курса национальной валюты [2]. Эта модель основана на тождестве национальных счетов и по своей методологической основе и структуре близка к модели кривых IS - LM, основы которой были заложены Д.Р. Хиксом [6]. По существу, эта модель представляет собой развитие хиксианской модели равновесия для условий открытой экономики. Р.А. Манделл предполагает наличие целого набора возможных сочетаний ставки процента и реального курса, способных обеспечить только внутреннее или внешнее равновесие и только одного сочетания, которое обеспечивает внутреннее и внешнее равновесие одновременно. В линейной форме, с использованием обозначений, принятых при рассмотрении модели кривых IS - LM, условие внутреннего равновесия могут быть записаны в виде следующего выражения:

$$
\mathrm{I}^{\prime}(\mathrm{i}) \mathrm{i}+\mathrm{NX}^{\prime}(\varepsilon) \varepsilon=\mathrm{I}_{0}+\mathrm{NX}_{0}-\mathrm{S}
$$

Где $\mathrm{I}_{0}$ и $\mathrm{NX}_{0}$ - максимальные значения объема инвестиций и чистого экспорта, соответствующие нулевым значениям процентной ставки и

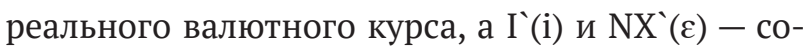
ответствующие линейные производные. Если бы объем национального производства мгновенно приспосабливался к объему совокупного спроса, то внутреннее равновесие поддерживалось бы автоматически при любых значениях ставки процента и реального обменного курса. Однако, поскольку этого не происходит, внутреннее равновесие обеспечивается только определенными сочетаниями значений ставки процента и реального курса, обращающими это уравнение в верное равенство. При этом предполагается, что объем денежной массы, а также величина государственных расходов и налогов остаются неизменными в течение рассматриваемого периода времени.

Условие внешнего равновесия может быть записано как:

$$
-\mathrm{NXK}^{\prime}(\mathrm{i}) \mathrm{i}+\mathrm{NX}^{\prime}(\varepsilon) \varepsilon=\mathrm{NX}_{0}-\mathrm{NXK}_{0}
$$

где $\mathrm{NXK}_{0}$ - максимум чистого экспорта капитала, при нулевой ставке процента, а NXK`(i) - линейная производная функции чистого экспорта капитала по величине ставки процента. Предполагается, что среднемировой уровень цен и ставки процента неизменны, поэтому «...условия торговли (реальный валютный курс - М.л.) могут изменяться лишь в результате колебаний обменного курса или вариаций внутренних цен» [2, с. 121].

Эти два уравнения можно объединить в линейную систему, которая имеет единственное экономически значимое решение, в случае если верно неравенство: I`(i) > NXK'(i) и $\mathrm{NX}_{0}>\mathrm{NXK}_{0}$, то есть эластичность инвестиций по проценту превышает эластичность чистого экспорта капитала по проценту, а максимальное значение 
чистого экспорта товаров и услуг должно быть выше максимального значения чистого экспорта капитала. В этом случае существует точка равновесия, которой соответствуют положительные значения процентной ставки и реального обменного курса, обеспечивающие установление внутреннего и внешнего равновесия.

Условия устойчивости равновесия Р.А. Манделл исследует, преобразуя исходную систему в систему дифференциальных уравнений, описывающих движение параметров системы во времени:

$$
\begin{aligned}
& \frac{d \varepsilon}{d t}=\left(k_{1}+h_{1}\right) N X^{\prime}(\varepsilon)\left(\varepsilon-\varepsilon_{0}\right)+ \\
& +\left(k_{1} I^{\prime}(i)+h_{1} N X K^{\prime}(i)\right)\left(i-i_{0}\right)
\end{aligned}
$$

$$
\begin{aligned}
& \frac{d i}{d t}=\left(h_{2}-k_{2}\right) N X^{\prime}(\varepsilon)\left(\varepsilon-\varepsilon_{0}\right)+ \\
& +\left(h_{2} I^{\prime}(i)-k_{2} N X K^{\prime}(i)\right)\left(i-i_{0}\right)
\end{aligned}
$$

где $\frac{d \varepsilon}{d t}$ и $\frac{d i}{d t}-$ скорости изменения адаптационных параметров системы - реального курса национальной валюты и ставки процента, $\mathrm{k}_{1}$ и $\mathrm{h}_{2}$ - скорости с которыми состояние внутреннего рынка отражается на величине, соответственно, реального курса и ставки процента, $\mathrm{k}_{2}$ и $\mathrm{h}_{1}-$ скорости с которыми состояние платежного баланса отражается на величине, соответственно, ставки процента и реального курса, $\varepsilon$ и $\mathrm{i}$ - текущие значения реального курса и ставки процента, $\varepsilon_{0}$ и $\mathrm{i}_{0}-$ равновесные значения реального курса и ставки процента. Разности текущих и равновесных значений представляют изменения адаптационных параметров системы, необходимые для приближения к равновесию.

Решение этой системы определяет условия, при которых отклонения адаптационных параметров системы от равновесных значений вызывают обратное движение к равновесию. Это будет происходить, если положительным значениям отклонений будут соответствовать отрицательные производные адаптационных параметров по времени и наоборот. Так что условия устойчивости являются определимыми [2, с. 146].

Движение «открытой экономики» к равновесию

Р.А. Манделл, при разработке своей модели, главное внимание уделяет исследованию динамического процесса приближения экономики к состоянию внутреннего и внешнего равновесия. Этот процесс может протекать непрерывно и поступательно или циклически, то есть в процессе периодических колебаний, когда система то приближается к равновесию, то удаляется от него, но при этом в каждом последующем цикле масштаб отклонения сокращается. Условия цикличности или ацикличности движения системы к равновесию зависят от системы формирования валютного курса.

В системе фиксированного курса центральный банк должен поддерживать внешнее равновесие, проводя активную кредитно-денежную политику и изменяя уровень процентной ставки, а внутреннее равновесие будет поддерживаться за счет изменения внутреннего уровня цен. А в системе плавающего курса внешнее равновесие поддерживается за счет колебаний обменного курса валюты, а внутренне за счет изменения ставки процента.

В любом случае система достигает равновесия ациклически если оба адаптационных параметра - реальный курс национальной валюты и ставка процента, достигают своих равновесных значений в один и тот же момент времени, то есть их движение к равновесию происходит в течение одного и того же периода времени. В противном случае система будет приближаться к равновесию, проходя через целую серию циклических колебаний.

Главным фактором, определяющим циклический или ациклический характер приближения системы к равновесию как в системе с фиксированным, так и в системе с плавающим валютным курсом, является подвижность капитала или эластичность чистого экспорта капитала по ставке процента. Только в системе с фиксированным курсом высокая подвижность капитала является условием ациклического приближения системы к равновесию, а в системе с плавающим курсом она, наоборот, будет порождать циклические колебания. Поэтому в условиях плавающего курса стабильному приближению к равновесию будет содействовать низкая или даже нулевая подвижность капитала [2, с. 128-133, 144-145].

Модель Р.А. Манделла представляет собой, по существу, развитие модели IS-LM для условий открытой экономики. Она демонстрирует, при каких условиях экономическая систе- 
ма, соответствующая базовым предпосылкам кейнсианской теории, будет достигать равновесия в условиях открытости. Однако, при этом Р.А. Манделла предполагает наличие неких экзогенных параметров равновесия, которые, по всей видимости, заданы производственными возможностями экономической системы $[9 ; 10]$. Такой подход соответствует неоклассическим принципам макроэкономического анализа, в соответствие с которыми рыночная экономика обладает своей внутренней устойчивостью и способна гасить колебания, возникающие под воздействием внешних факторов и отклоняющих ее от параметров устойчивого равновесия. При таком подходе государственная система макроэкономического регулирования может рассматриваться исключительно как инструмент ускоренной адаптации экономики к параметрам устойчивого равновесия, который может при определенных условиях заменять естественные рыночные адаптационные механизмы, если их действие занимает слишком много времени.

\section{Библиографический список}

1. Блауг М. Экономическая мысль в ретроспективе. М.: Дело ЛТД.,1994.

2. Манделл Р.А. Динамика адаптации мирового валютного рынка при фиксированном и плавающем обменных курсах. // Этот изменчивый обменный курс.- М. 2001. С. 117-154.

3. Манделл Р.А. Выбор экономической политики для достижения внутреннего и внешнего равновесия. // Этот изменчивый обменный курс.- М. 2001. С. 155-164.

4. Патинкин Д. Деньги, процент и цены. Соединение теории денег и теории стоимости.- М., 2004.

5. Хансен Э. Экономические циклы и национальный доход. / Классики кейнсианства. Т. 1, 2. М.: Экономика, 1997. С. 202-415.; 1-431.

6. Хикс Д.Р. Господин Кейнс и классики: попытка интерпретации. // Истоки: вопросы истории народного хозяйства и экономической мысли. Вып. 3.- М.: ГУ-ВШЭ, 1998. С. 293-337.

7. Mundell R.A. Monetary theory; inflation, interest, and growth in the world economy. Pacific Palisades, Calif. 1971.

8. Prasch R.E. The Economic Contributions of Robert A. Mundell // Review of Political Economy. Volume 13, 2001. pp. 41-58.

9. Weeks J. Open Economy Monetary Policy Reconsidered // Review of Political Economy. Volume 25, 2013. pp. 57-67.

10. Sarantis N. The Mundell-Fleming Model with Perfect Capital Mobility and Oligopolistic Pricing // Journal of Post Keynesian Economics. Volume 9. 1986, pp. 138-148. 